Research Paper

\title{
Long non-coding RNA MSC-AS1 facilitates the proliferation and glycolysis of gastric cancer cells by regulating PFKFB3 expression
}

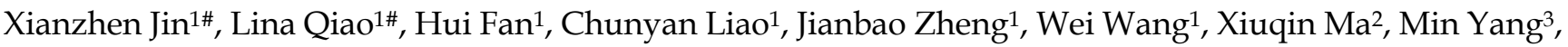 \\ Xuejun Sun ${ }^{1}$ and Wei Zhao ${ }^{\circledR}$ \\ 1. Department of General Surgery, The First Affiliated Hospital of Xi'an Jiaotong University, Xi'an 710061, P.R. China. \\ 2. Department of Nursing, Hanzhong Central Hospital, Hanzhong 723000, P.R. China. \\ 3. Department of Nursing, Xianyang Hospital, Yan'an University, Xianyang 712000, P.R. China. \\ \#These authors contributed equally to this work. \\ $\triangle$ Corresponding authors: Prof. Wei Zhao, Tel: +8602985323870, E-mail: zhaowei9803@xjtufh.edu.cn; and Prof. Xuejun Sun, Tel: +86029-85323874, E-mail: \\ sunxy@mail.xjtu.edu.cn. Department of General Surgery, The First Affiliated Hospital of Xi'an Jiaotong University, 277 Yanta West Road, Xi'an 710061, P.R. \\ China.
}

(c) The author(s). This is an open access article distributed under the terms of the Creative Commons Attribution License (https://creativecommons.org/licenses/by/4.0/). See http://ivyspring.com/terms for full terms and conditions.

Received: 2020.08.14; Accepted: 2020.11.19; Published: 2021.01.01

\begin{abstract}
Long non-coding RNA musculin antisense RNA 1 (IncRNA MSC-AS1) has been recognized as an oncogene in pancreatic cancer, hepatocellular carcinoma, nasopharyngeal carcinoma, and renal cell carcinoma. However, the functional significance of MSC-AS1 and its underlying mechanism in gastric cancer (GC) progression remain unclear. In this study, we demonstrated that the expression of MSC-AS1 in GC tissues was significantly higher than that in non-tumor tissues. Moreover, the elevated level of MSC-AS1 was detected in GC cells (MKN-45, AGS, SGC-7901, and MGC-803) compared to normal GES-1 gastric mucosal cells. The cancer genome atlas (TCGA) data further indicated that the high level of MSC-ASI was closely correlated with advanced tumor stage and poor prognosis of GC. Next, we revealed that MSC-AS1 knockdown inhibited the proliferation, glucose consumption, lactate production, and pyruvate production of MGC-803 cells. Conversely, MSC-AS1 overexpression enhanced the proliferation and glycolysis of AGC cells. Mechanistically, modulating MSC-ASI level affected the expression of 6-phosphofructo-2-kinase/fructose-2,6-biphosphatase 3 (PFKFB3), but did not impact the levels of hexokinase 2 (HK2) and pyruvate kinase M2 (PKM2) in GC cells. Based on this, we reversed the MSC-AS1 knockdown-induced the inhibition of cell proliferation and glycolysis by restoring PFKFB3 expression in MGC-803 cells. In conclusion, MSC-AS1 facilitated the proliferation and glycolysis of GC cells by maintaining PFKFB3 expression.
\end{abstract}

Key words: Gastric cancer; LncRNA; MSC-AS1; PFKFB3; Proliferation; Glycolysis

\section{Introduction}

Gastric cancer (GC), one of the most common malignancies, has a high morbidity and mortality worldwide [1]. Currently, surgical resection remains the leading therapeutic strategy for GC [2]. Chemotherapy, targeted therapy, and immunotherapy are preferred options for advanced GC [2]. But, the clinical outcomes of GC patients have not shown satisfactory improvement $[3,4]$. Thus, it is essential to investigate the underlying mechanisms involved in GC's initiation and progression and explore novel therapeutic targets for GC.

Previous studies have demonstrated that the human genome encodes long non-coding RNAs (lncRNAs; $\geq 200$ nucleotides in length), which have a limited or no ability to encode proteins [5]. Currently, increasing evidence supports that lncRNAs act as vital regulators in cellular development, differentiation, and various other biological processes [6]. Importantly, lncRNAs perform critical functions in the initiation and progression of human cancers 
[7-14]. For instance, lncRNA PVT1 is highly expressed in GC and prominently correlated with patients' poor clinical outcomes [15]. PVT1 directly binds to signal transducer and activator of transcription 3 (STAT3) and maintains its protein stability in GC cells [15]. The overexpressed lncRNA HOXC-AS3 promotes GC cells' proliferation and migration by interacting with Y-box binding protein 1 (YBX1) [16]. Besides, lncRNA MACC1-AS1 enhances GC cells' proliferation and glycolysis via the AMPK/Lin28 pathway-mediated MACC1 upregulation [17]. LncRNA musculin antisense RNA 1 (MSC-AS1) has been recognized as an oncogene in pancreatic ductal adenocarcinoma (PDAC) [18], hepatocellular carcinoma (HCC) [19], nasopharyngeal carcinoma (NPC) [20], and renal cell carcinoma (RCC) [21]. MSC-AS1 functions as a molecular sponge for miR-29b-3p to increase cyclindependent kinase 14 (CDK14) expression, which facilitates PDAC cell proliferation [18]. MSC-AS1 contributes to cell proliferation and invasion in RCC via activating the miR-3924-mediated Wnt/ $\beta$-catenin signaling pathway [21]. MSC-AS1 is reported to promote the proliferation, apoptosis resistance, invasion, and epithelial-to-mesenchymal transition (EMT) of NPC cells by regulating the miR-524-5p/ nuclear receptor subfamily 4 group A member 2 (NR4A2) axis [20]. Moreover, MSC-AS1 accelerates HCC progression through enhancing the expression of phosphoglycerate kinase 1 [19]. However, it is unclear whether and how MSC-AS1 participates in GC progression.

In the present study, we determined the expression difference of MSC-AS1 between GC and noncancerous tissues. The clinical significance of MSC-AS1 in GC was analyzed according to the cancer genome atlas (TCGA) data. Then, we explored the effects of MSC-AS1 on GC cell proliferation and glycolysis and investigated its possible underlying mechanism. Our data suggested that MSC-AS1 was highly expressed in GC and facilitated cell proliferation and glycolysis by enhancing 6-phosphofructo-2-kinase/fructose-2,6-biphosphatase 3 (PFKFB3) expression.

\section{Materials and methods}

\section{Human tissue samples}

Eighty GC tissue samples and 80 tumor-adjacent tissue samples were obtained from patients who signed informed consent forms and received surgical resection at $1^{\text {st }}$ Affiliated Hospital of Xi'an Jiaotong University. All specimens were pathologically confirmed as GC and stored at $-80^{\circ} \mathrm{C}$ for subsequent analysis. This study was approved by the Ethics Committee of the First Affiliated Hospital of Xi'an
Jiaotong University. The clinical features of the patients were described in our previous study [22].

\section{Cell culture and transfection}

The human GC cells (MKN-45, AGS, SGC-7901, and MGC-803) and normal GES-1 gastric mucosal cells were maintained in our lab under standard culture conditions [22]. MSC-AS1 shRNA (shMSCAS1-1 and shMSC-AS1-2) and non-targeting RNA (NT shRNA) were obtained from GenePharma (Shanghai, China). The pcDNA3.1-PFKFB3 and pcDNA3.1-MSC-AS1 vectors were constructed by inserting the cDNA products of PFKFB3 and MSC-AS1 into the expression vector pcDNA3.1 (Invitrogen, Carlsbad, CA, USA). The plasmids were delivered into GC cells using Lipofectamine 2000 (Invitrogen) according to the manufacturer's instructions.

\section{Real-time quantitative PCR (qRT-PCR)}

Total RNAs were isolated from tissues and cells with TRIzol reagent (Invitrogen), and reversetranscribed into cDNA using the TIANScript RT Kit (Tiangen Biotech, Beijing, China). qRT-PCR was performed using SYBR Green PCR Master Mix (Takara, Dalian, China) in the CFX96 Touch ${ }^{\mathrm{TM}}$ RealTime PCR Detection System (Bio-Rad Laboratories, Hercules, CA, USA). The expression level of mRNA was normalized to GAPDH expression using the $2^{-\Delta \Delta \mathrm{Ct}}$ method. The sequences of the primers were listed as follows. MSC-AS1 forward 5'-AAGCAACAA CTGTCTGGCCT-3', reverse 5'-TGATGCCAGCAAAT TGGTGC-3'; 6-phosphofructo-2-kinase/fructose-2,6biphosphatase 3 (PFKFB3) forward 5'- CAGTTGTGG CCTCCAATATC-3', reverse 5'- GGCTTCATAGCAA CTGATCC-3'; hexokinase 2 (HK2) forward 5'- ATTGT CCAGTGCATCGCGGA-3', reverse 5' - AGGTCAAAC TCCTCTCGCCG-3'; pyruvate kinase M2 (PKM2) forward 5'-CAGAGGCTGCCATCTACCAC-3', reverse 5'-CCAGACTTGGTGAGGACGAT-3'; GAPDH forward 5'-AGCAAGAGCACAAGAGG AAG-3', reverse 5'-GGTTGAGCACAGGGTACTTT-3'.

\section{Cell proliferation analysis}

The proliferation ability of transfected cells was analyzed using the Cell Counting Kit-8 (CCK-8; Dojindo Laboratories, Dojindo, Japan) assay and 5-ethynyl-2'-deoxyuridine (EdU) staining. For CCK-8 assay, the transfected cells $\left(2 \times 10^{3}\right.$ cells per well $)$ were seeded into 96 -well plates. $10 \mu \mathrm{L}$ of CCK-8 reagent was added into the plates and incubated for an additional $2 \mathrm{~h}$ at $37^{\circ} \mathrm{C}$. Optical-density values at 450 $\mathrm{nm}$ were measured with a microplate reader (Thermo Fisher Scientific, Waltham, MA, USA). The Cell-Light ${ }^{\mathrm{TM}}$ EdU Apollo®488 In vitro Imaging Kit 
(RIBOBIO, Guangzhou, China) was used for EdU staining, as previously described [23].

\section{Detection of Glucose consumption, lactate production, and pyruvate production}

The transfected cells $\left(1 \times 10^{6}\right.$ cells per well) were seeded into 6-well plates and incubated for $24 \mathrm{~h}$ at 37 ${ }^{\circ} \mathrm{C}$ under indicated treatment. The culture medium was collected and subjected to a glucose assay kit (ab136955, Abcam, Cambridge, MA, USA), lactate assay kit (ab65331, Abcam), and pyruvate assay kit (ab65342, Abcam) for measuring glucose consumption, lactate production, and pyruvate production according to the manufacturer's instructions.

\section{Western blotting}

Cells were lysed with the RIPA lysis buffer (Beyotime, Shanghai, China) containing a proteasome inhibitor (PI, Roche, Indianapolis, IN, USA) and a phosphatase inhibitor (Thermo Fisher Scientific, Waltham, MA, USA). $20 \mu \mathrm{g}$ of each protein were resolved by SDS-PAGE and transferred to PVDF membranes (Millipore, Bedford, MA, USA). The membranes were blocked with $5 \%$ skimmed milk for $1 \mathrm{~h}$ at room temperature and then incubated with the PFKFB3 antibody (ab181861, Cambridge, MA, USA) and GAPDH antibody (ab8226, Abcam) at $4{ }^{\circ} \mathrm{C}$ overnight. The next day, we incubated the membranes with suitable horseradish peroxidase (HRP)-conjugated secondary antibody (Beyotime) for $1 \mathrm{~h}$ at room temperature. The blots were then visualized with the ECL reagent (Millipore) and detected by an Amersham Imager 600 (GE Healthcare Life Sciences, Pittsburgh, PA, USA). The ImageJ software (1.46; National Institutes of Health, Bethesda, MD, USA) was used for semi-quantitative immunoblots analysis.

\section{Statistical analysis}

The data of at least three independent experiments were presented as the mean $\pm \mathrm{SD}$ and analyzed with GraphPad Prism 8.0 (GraphPad Inc., San Diego, CA, USA). The one-way ANOVA and Student's $t$-test were performed to calculate the differences among the groups. $P<0.05$ was considered significant.

\section{Results}

\section{The elevated level MSC-ASI associated with the poor prognosis of $G C$}

First, qRT-PCR was carried out to determine the expression difference of MSC-AS1 between GC and adjacent nontumor tissues. Our results showed that the level of MSC-AS1 in GC tissue samples was significantly higher than that in the tumor-adjacent tissue samples $(P<0.0001$, Figure $1 \mathrm{~A})$. Moreover, TCGA data from the gene expression profiling interactive analysis (GEPIA) platform [24] and gene expression omnibus (GEO) datasets (GSE54129, GSE65801, and GSE13911) from lnCAR platform [25] consistently revealed the upregulated expression of MSC-AS1 in GC tissues compared to normal tissues $(P<0.05$, Figure $1 \mathrm{~B}$ and Supplementary Figure 1$)$. MSC-AS1 expressed at higher levels in GC cells (MKN-45, AGS, SGC-7901, and MGC-803) as compared with normal GES-1 gastric mucosal cells $(P<0.05$, Figure 1C). Notably, the analysis of TCGA data using GEPIA platform indicated that a high MSC-AS1 level in GC tissues was correlated with advanced tumor stage and predicted poor prognosis for the patients $(P<0.05$, Figure $1 \mathrm{D}$ and $1 \mathrm{E})$.

\section{MSC-ASI affected the proliferation and glycolysis of GC cells}

Next, we investigated the proliferation and glycolysis of GC cells after modulating the MSC-AS1 level. The expression of MSC-AS1 was significantly knocked down in MGC-803 cells, which expressed the relative highest level of MSC-AS1, using two independent shRNAs $(P<0.05$, Figure $2 \mathrm{~A})$. The CCK-8 assay revealed that MGC-803 cells' viability was markedly suppressed by MSC-AS1 knockdown $(P<0.05$, Figure 2B). The proliferation of MGC-803 cells was prominently decreased by MSC-AS1 silencing, as suggested by EdU staining $(P<0.05$, Figure 2C). Furthermore, the depletion of MSC-AS1 remarkably reduced glucose consumption, lactate production, and pyruvate production in MGC-803 cells $(P<0.05$, Figure 2D-2F). Then, MSC-AS1 was overexpressed in AGS cells, which expressed the relative lowest level of MSC-AS1, using the expression plasmid $(P<0.05$, Figure $3 \mathrm{~A})$. Conversely, MSC-AS1 overexpression significantly enhanced the proliferation and glycolysis of AGS cells $(P<0.05$, Figure 3B-3F).

\section{MSC-AS1 regulated PFKFB3 expression in GC cells}

KEGG pathway analysis of GEO) datasets (GSE54129, GSE65801, and GSE13911) from lnCAR platform suggested the involvement of MSC-AS1 in GC's metabolic pathways (Supplementary Figure 2). Since glycolysis is regulated by three key rate-limiting enzymes, including HK2, PFKFB3, and PKM2 [26]. Thus, the correlations between MSC-AS1 and HK2 mRNA, PFKFB3 mRNA, and PKM2 mRNA were determined in GC tissues from TCGA database using GEPIA platform. Our data found that MSC-AS1 was positively correlated with PFKFB3 mRNA in GC 
tissues $(P<0.0001$, Figure $4 \mathrm{~A})$, but did not associate with HK2 mRNA and PKM2 mRNA (Supplementary Figure 3). Then, the levels of HK2 mRNA, PFKFB3 mRNA, and PKM2 mRNA were detected in GC cells after modulating MSC-AS1 expression. As shown in Figure 4B and 4C, MSC-AS1 knockdown decreased the PFKFB3 mRNA level in MGC-803 cells, while MSC-AS1 overexpression increased the expression of PFKFB3 mRNA in AGS cells $(P<0.05)$. However, MSC-AS1 did not impact HK2 mRNA and PKM2 mRNA levels in GC cells (Figure 4B and 4C). Consistently, MSC-AS1 positively regulated PFKFB3 protein expression in GC cells as suggested by western blotting analysis $(P<0.05$, Figure $4 \mathrm{D}$ and $4 \mathrm{E})$. TCGA database analysis using GEPIA platform indicated that PFKFB3 mRNA expression in GC tissues was significantly higher than that in normal tissues $(P<0.0001$, Supplementary Figure 4A). Moreover, PFKFB3 mRNA expressed at higher levels in GC cells (MKN-45, AGS, SGC-7901, and MGC-803) as compared with GES- 1 cells $(P<0.05$, Supplementary Figure 4B).
PFKFB3 mediated the role of MSC-AS1 in GC cells

The expression of PFKFB3 was restored in MSC-AS1 knockdown MGC-803 cells $(P<0.05$, Figure $5 \mathrm{~A})$ to confirm whether PFKFB3 was a downstream effector of MSC-AS1. A CCK-8 analysis indicated that the viability of MSC-AS1 silenced MGC-803 cells was significantly promoted by PFKFB3 restoration $(P<0.05$, Figure 5B). Besides, PFKFB3 restoration markedly reversed MSC-AS1 knockdown-induced the proliferation inhibition in MGC-803 cells $(P<0.05$, Figure 5C). Significantly, MSC-AS1 silencing-reduced glucose consumption, lactate production, and pyruvate production were prominently enhanced by the re-expression of PFKFB3 in MGC-803 cells $(P<0.05$, Figure 5D-5F).

\section{Discussion}

The aberrant level of lncRNAs has been detected in GC tissues and serum samples, which may be used as potential diagnostic and prognostic biomarkers [27]. For example, the high expression levels of
A

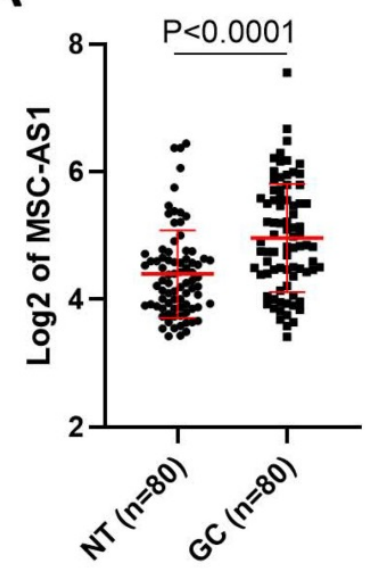

D

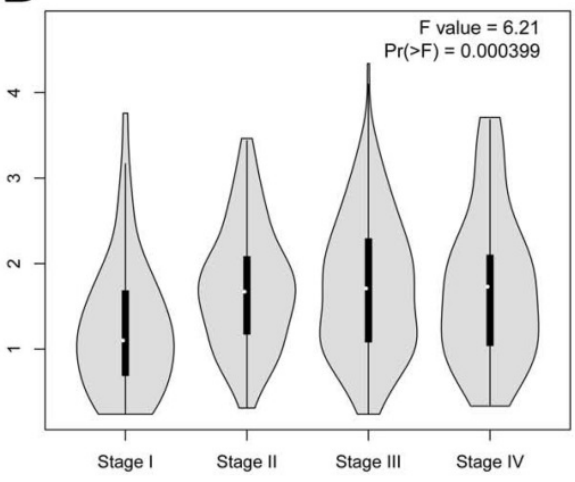

B
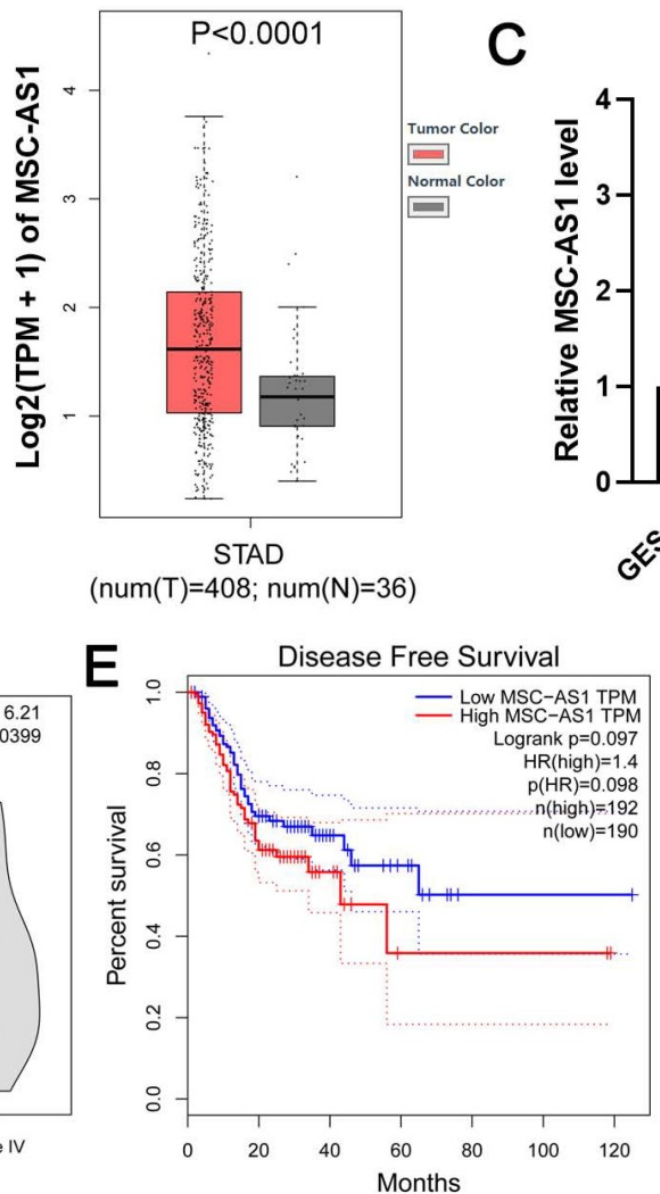

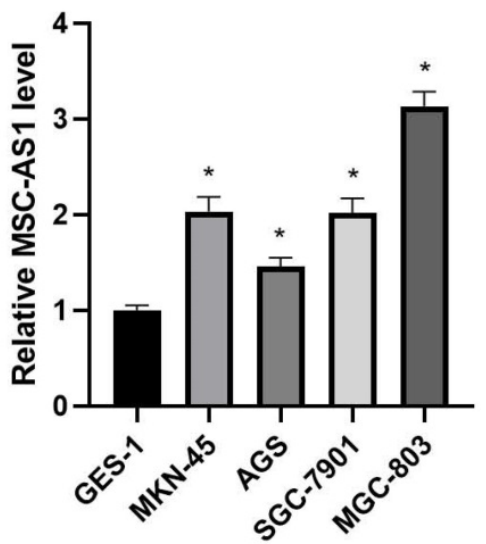

Figure 1. The expression and prognostic significance of MSC-AS1 in GC. (A) The expression of MSC-AS1 in 80 pairs of GCs and adjacent nontumor (NT) tissues. (B) CGA data from GEPIA platform revealed an upregulated expression of MSC-AS1 in GC tissues. (C) The levels of MSC-AS1 in GC cells (MKN-45, AGS, SGC-7901, and MGC-803) were compared with normal GES-1 gastric mucosal cells. (D) TCGA data from the GEPIA platform indicated that the high level of MSC-ASI correlated with GC's advanced tumor stage. (E) TCGA data from the GEPIA platform suggested that GC patients with an increased expression of MSC-AS1 in tumor tissues had an apparent lower overall survival and disease-free survival. $* \mathrm{P}<0.05$. 
lncRNA HOXC-AS3 are correlated with lower overall survival in GC patients, as demonstrated by KaplanMeier survival analysis [16]. Compared to carcinoembryonic antigen, the exosomal lncUEGC1 in the serum shows a higher diagnostic accuracy for discriminating GC patients from healthy individuals [28]. The expression of MSC-AS1 is frequently overexpressed in PDAC, HCC, RCC, and NPC [18-21]. Moreover, the high level of MSC-AS1 predicts poor clinical outcomes of patients with PDAC, HCC, and RCC $[18,19,21]$. Here, we revealed an upregulated expression of MSC-AS1 in GC tissues and cells. TCGA data and GEO data further supported the highly expressed MSC-AS1in GC. Interestingly, TCGA data demonstrated that the elevated expression of MSC-AS1 was correlated with advanced tumor stage and poor prognosis of GC. Therefore, our results suggested MSC-AS1 as a promising prognostic marker in GC.

Previous studies have explored the biological function of MSC-AS1 in human cancers, including PDAC, HCC, RCC, and NPC [18-21]. MSC-AS1 knockdown induces proliferation inhibition, apoptosis, and G1 phase arrest, and represses cell migration/invasion in HCC cells [19]. MSC-AS1 participates in tumor progression via promoting cell proliferation and invasion in NPC and RCC $[18,20]$. Also, MSC-AS1 silencing significantly suppressed the proliferation of PDAC cells [18]. In this study, loss-of and gain-of-function experiments indicated that MSC-AS1 positively regulated cell proliferation, glucose consumption, lactate production, and pyruvate production in GC cells. Thus, MSC-AS1 acted as an oncogene in GC.
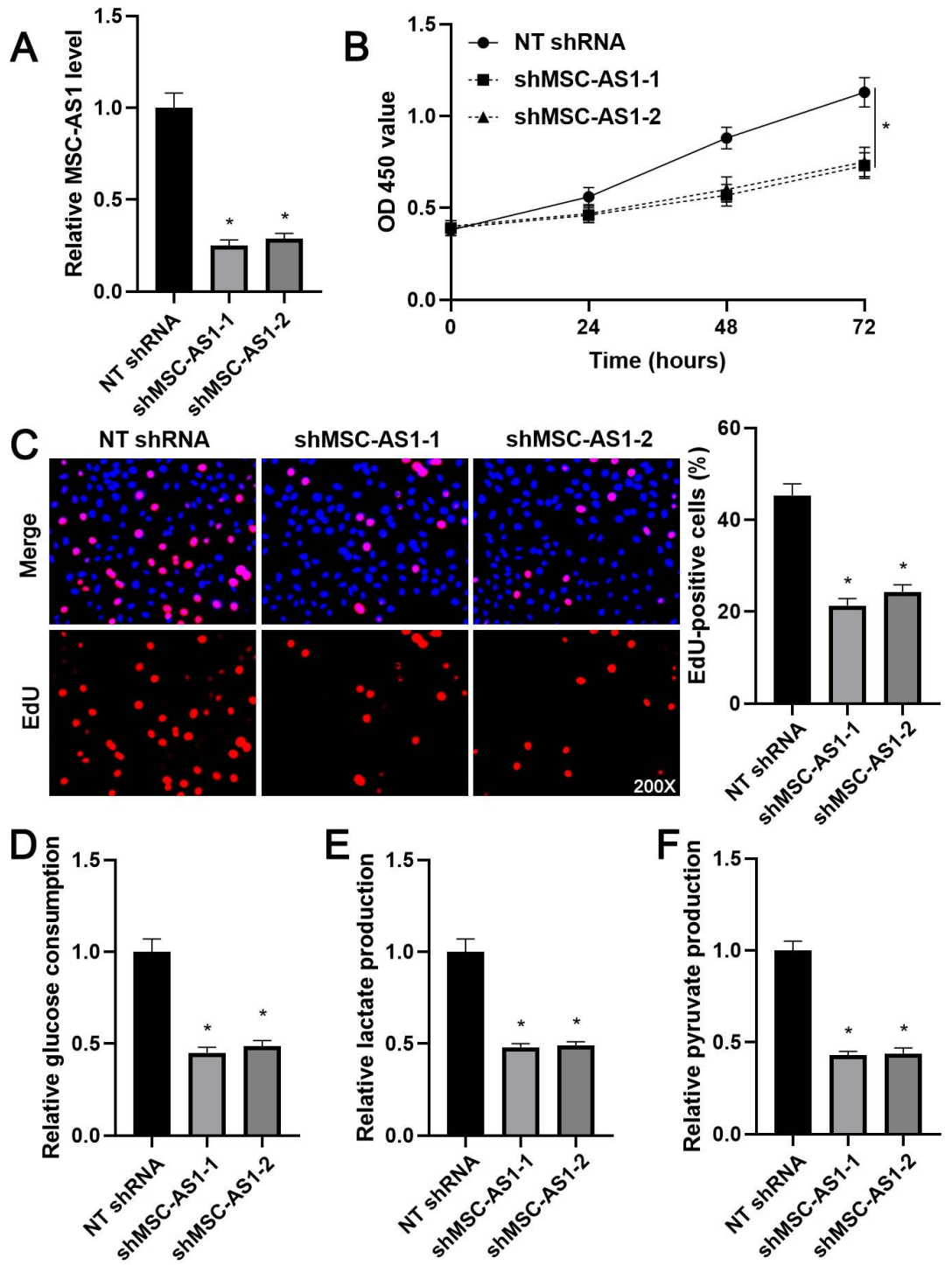

Figure 2. MSC-AS1 knockdown inhibits the proliferation and glycolysis of MGC-803 cells. (A) MGC-803 cells were transfected with non-targeting (NT) shRNA or MSC-ASI shRNAs (shMSC-AS1-1 and shMSC-AS1-2) and assessed by qRT-PCR for MSC-AS1 expression. (B) A CCK-8 assay indicated that MSC-AS1 knockdown inhibited the viability of MGC-803 cells. (C) An EdU staining revealed that MSC-AS1 silencing repressed the proliferation of MGC-803 cells. (D-F) The glucose consumption, lactate production, and pyruvate production were decreased after MSC-AS1 knockdown in MGC-803 cells. *P<0.05. 

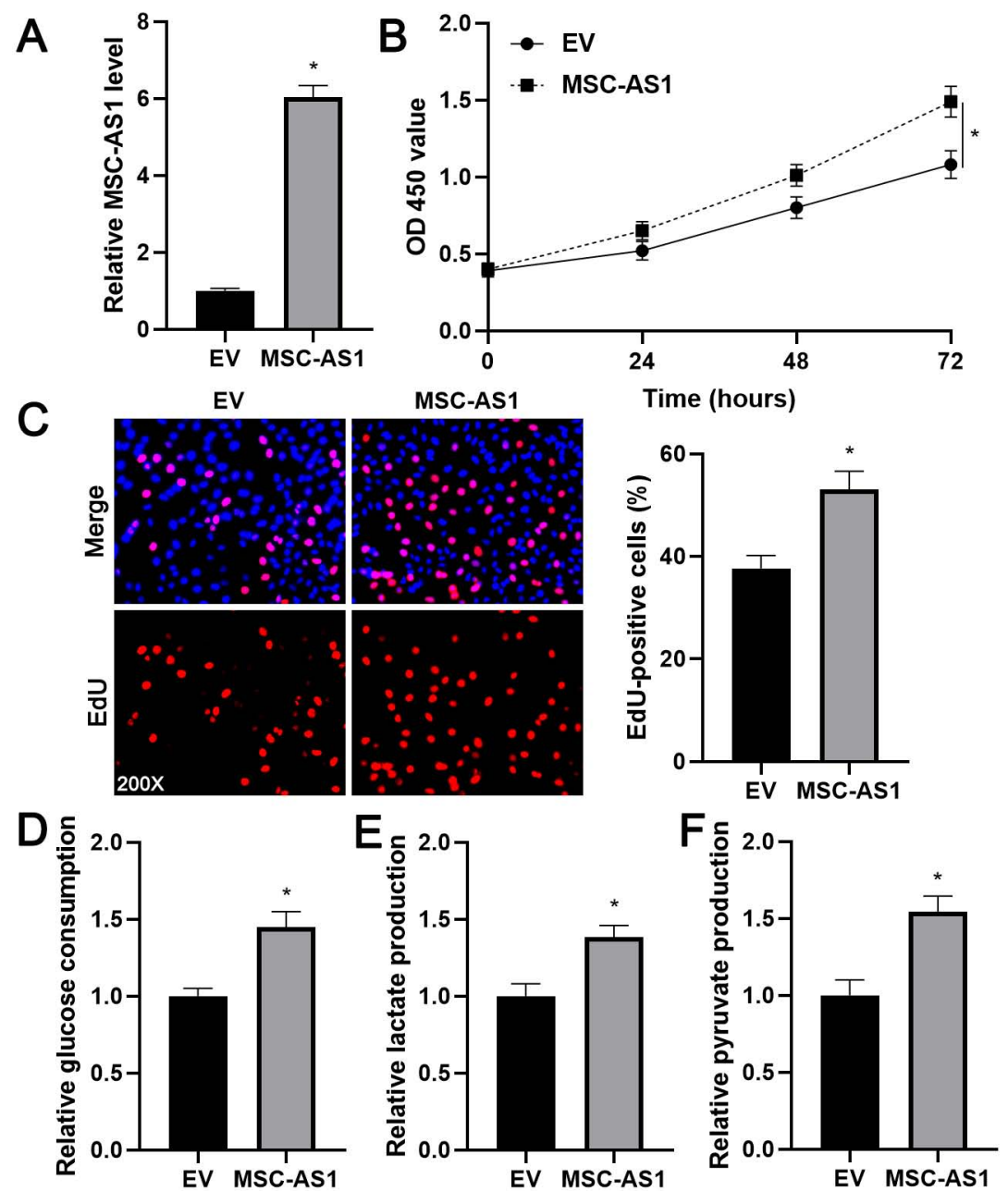

Figure 3. MSC-ASI overexpression promotes the proliferation and glycolysis of AGS cells. (A) AGS cells were transfected with empty vector (EV) or pcDNA3.1-MSC-AS1 and assessed by qRT-PCR for MSC-AS1 expression. (B) A CCK-8 assay indicated that MSC-AS1 overexpression promoted the viability of AGS cells. (C) An EdU staining revealed that MSC-ASI silencing facilitated the proliferation of AGS cells. (D-F) The glucose consumption, lactate production, and pyruvate production were enhanced after MSC-AS1 overexpression in AGS cells. $* \mathrm{P}<0.05$.

Glycolysis, also known as the Warburg effect, is an intracellular metabolic process consisting of 10 consecutive enzymatic reactions that degrade one molecule of glucose into two pyruvates [29]. Enhance glycolysis supports cancer cells' rapid growth, provides essential precursors for cell biosynthesis, and promotes tumor angiogenesis [26]. HK2, PFKFB3, and PKM2 are identified initially as key glycolysis enzymes that participate in glucose metabolism [26, 30]. A recent study reports that lncRNA AGPG enhances glycolysis activity and promotes tumor growth via maintaining PFKFB3 protein stability in esophageal squamous cell carcinoma (ESCC). Here, our results suggested that MSC-AS1 regulated PFKFB3 expression at the mRNA level. We found that MSC-AS1 enhanced the expression of PFKFB3 mRNA and protein in GC cells, but did not impact the levels of HK2 and PKM2. TCGA data also revealed the positive correlation between MSC-AS1 and PFKFB3
mRNA expression in GC tissues. Prior studies have demonstrated that PFKFB3 is highly expressed in GC and facilitated the proliferation, migration, and invasion of cancer cells [31, 32]. After that, the role of PFKFB3 in the metabolic control of cancer cell glycolysis has been widely reported [33-35]. Hypoxia-induced PFKFB3 and PFKFB4 play a vital role in the Warburg effect in GC cells [36]. Our study further found that PFKFB3 restoration significantly enhanced the knockdown of MSC-AS1-attenuated GC cells' proliferation and glycolysis.

Collectively, our data explored the overexpression of MSC-AS1 in GC. Moreover, we demonstrated the significant roles of MSC-AS1 in GC cell proliferation and glycolysis. PFKFB3, which was positively regulated by MSC-AS1, mediated the role of MSC-AS1 in GC cells. Future work is needed to uncover the underlying mechanism involved in regulating PFKFB3 by MSC-AS1 in GC. 

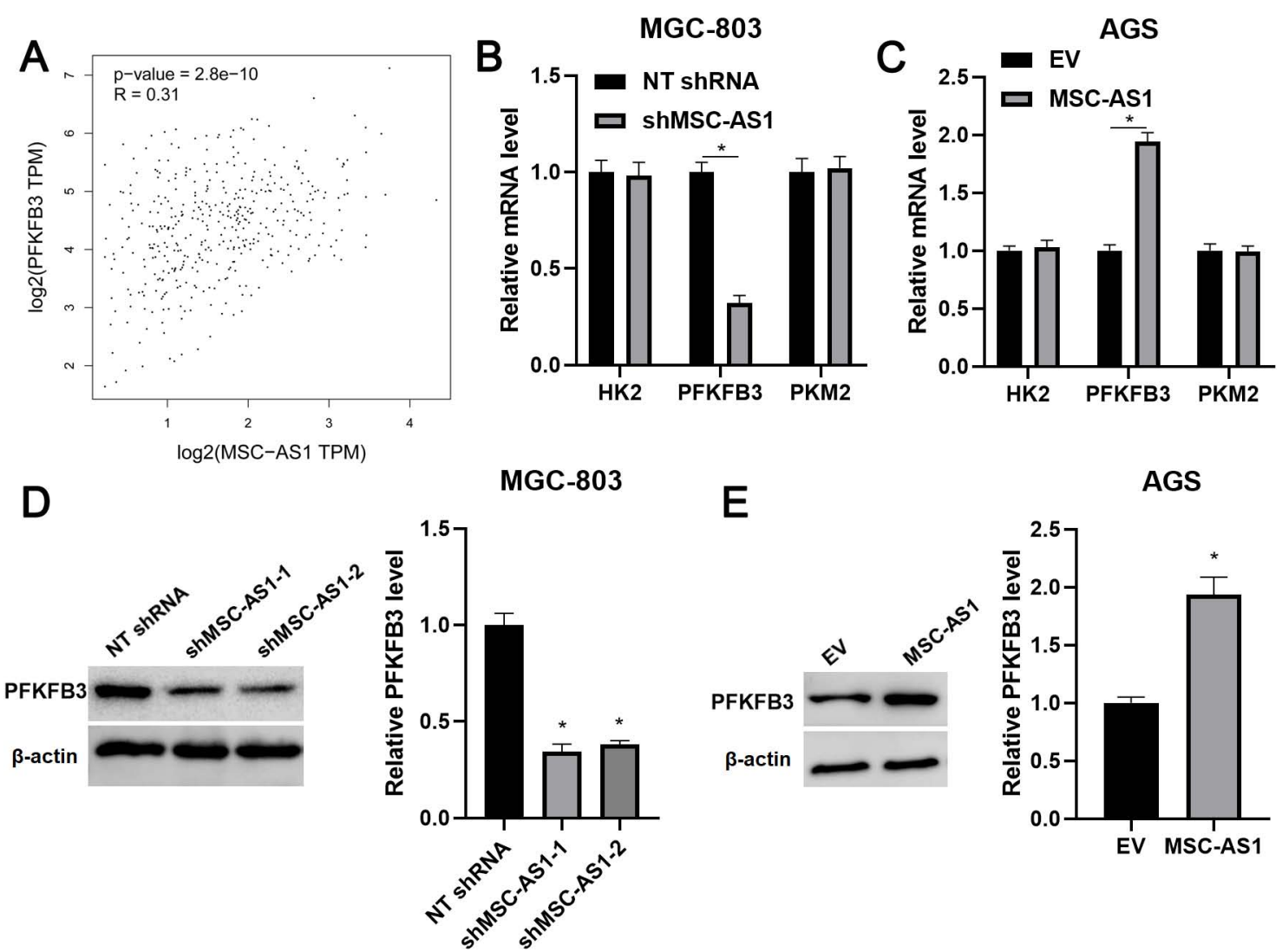

Figure 4. MSC-AS1 regulates PFKFB3 expression in GC cells. (A) TCGA data from the GEPIA platform indicated a positive correlation between MSC-AS1 and PFKFB3 mRNA expression in GC tissues. (B) MGC-803 cells were transfected with non-targeting (NT) shRNA or MSC-AS1 shRNAs (shMSC-AS1-1 and shMSC-AS1-2) and assessed by qRT-PCR for HK2, PFKFB3, and PKM2 mRNA expression. (C) AGS cells that were transfected with empty vector (EV) or pcDNA3.1-MSC-AS1 and assessed by qRT-PCR for HK2, PFKFB3, and PKM2 mRNA expression. (D) MSC-AS1 knockdown reduced the level of PFKFB3 protein in MGC-803 cells. (E) MSC-AS1 overexpression increased the expression of PFKFB3 protein in AGS cells. $* P<0.05$
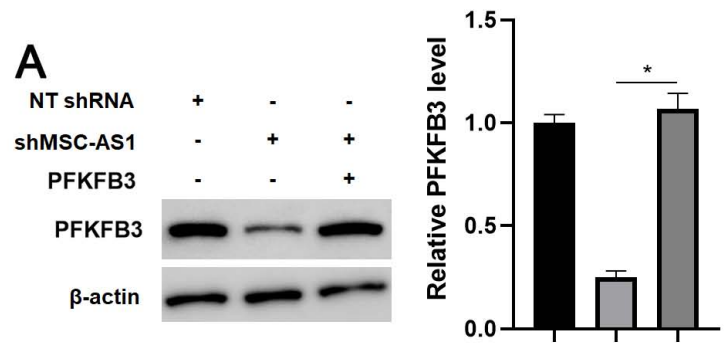

$$
\text { NT ShRNA + }
$$

ShMSC-AS1 - + +

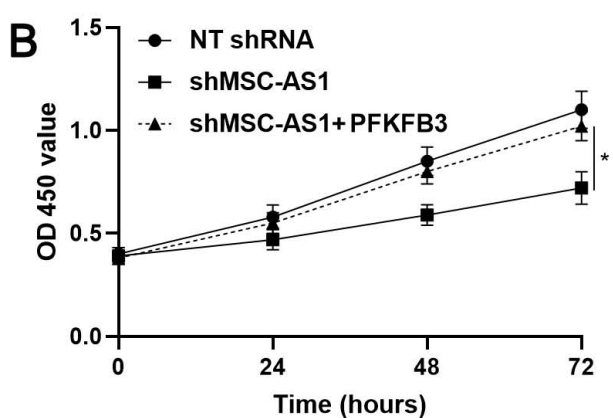

PFKFB3 - - + +
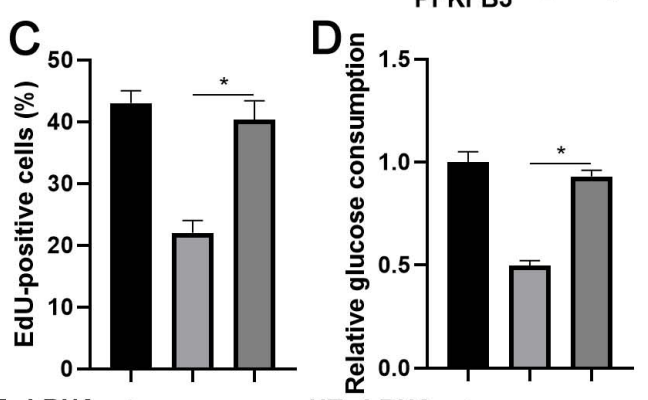

E
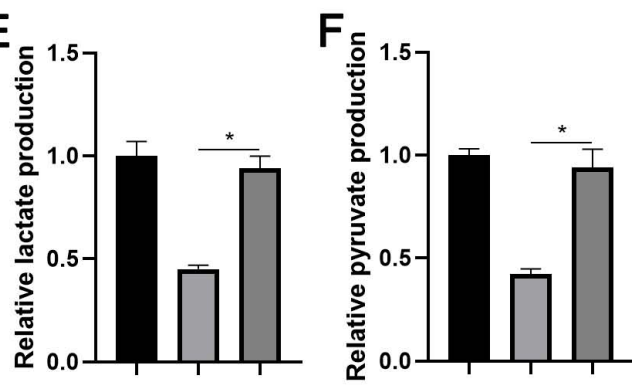

NT ShRNA +

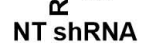

NT ShRNA

shMSC-AS1

shMSC-AS1

shMSC-AS1

PFKFB3

Figure 5. PFKFB3 restoration reverses the effects of MSC-AS1 knockdown on MGC-803 cells. (A) MSC-AS1 knockdown MGC-803 cells were transfected with pcDNA3.1-PFKFB3 to restore PFKFB3 expression. (B) CCK-8, (C) EdU, (D) glucose consumption, (E) lactate production, and (F) pyruvate production assays were performed to detect the proliferation and glycolysis of MGC-803 cells. $* \mathrm{P}<0.05$. 


\section{Abbreviations}

GC, gastric cancer; LncRNA, long non-coding RNA; STAT3, signal transducer and activator of transcription 3; YBX1, Y-box binding protein 1; MSC-AS1, musculin antisense RNA 1; PDAC, pancreatic ductal adenocarcinoma; HCC, hepatocellular carcinoma; NPC, nasopharyngeal carcinoma; RCC, renal cell carcinoma; CDK14, cyclin dependent kinase 14; EMT, epithelial-tomesenchymal transition; NR4A2, nuclear receptor subfamily 4 group A member 2; PFKFB3, 6-phosphofructo-2-kinase/fructose-2,6-biphosphatase 3; HK2, hexokinase 2; PKM2, pyruvate kinase M2; ESCC, esophageal squamous cell carcinoma.

\section{Supplementary Material}

Supplementary figures. http://www.medsci.org/v18p0546s1.pdf

\section{Acknowledgements}

This work was supported by the grant from the Key Research and Development Program of Shaanxi (2019SF-057).

\section{Competing Interests}

The authors have declared that no competing interest exists.

\section{References}

1. Bray F, Ferlay J, Soerjomataram I, Siegel RL, Torre LA, Jemal A. Global cancer statistics 2018: GLOBOCAN estimates of incidence and mortality worldwide for 36 cancers in 185 countries. CA Cancer J Clin. 2018; 68: 394-424.

2. Patel TH, Cecchini M. Targeted Therapies in Advanced Gastric Cancer. Curr Treat Options Oncol. 2020; 21: 70.

3. Canale M, Casadei-Gardini A, Ulivi P, Arechederra M, Berasain C, Lollini PL, et al. Epigenetic Mechanisms in Gastric Cancer: Potential New Therapeutic Opportunities. Int J Mol Sci. 2020; 21

4. Mungan I, Dicle CB, Bektas S, Sari S, Yamanyar S, Cavus M, et al. Does the preoperative platelet-to-lymphocyte ratio and neutrophil-to-lymphocyte ratio predict morbidity after gastrectomy for gastric cancer? Mil Med Res. 2020; 7: 9.

5. Guttman M, Rinn JL. Modular regulatory principles of large non-coding RNAs. Nature. 2012; 482: 339-46.

6. Orom UA, Derrien T, Beringer M, Gumireddy K, Gardini A, Bussotti G, et al. Long noncoding RNAs with enhancer-like function in human cells. Cell. 2010; 143: 46-58

7. Wang Y, Yang L, Chen T, Liu X, Guo Y, Zhu Q, et al. A novel lncRNA MCM3AP-AS1 promotes the growth of hepatocellular carcinoma by targeting miR-194-5p/FOXA1 axis. Mol Cancer. 2019; 18: 28.

8. Wang Y, Liu Z, Yao B, Li Q, Wang L, Wang C, et al. Long non-coding RNA CASC2 suppresses epithelial-mesenchymal transition of hepatocellular carcinoma cells through CASC2/miR-367/FBXW7 axis. Mol Cancer. 2017; 16: 123.

9. Zhang L, Wang L, Wang Y, Chen T, Liu R, Yang W, et al. LncRNA KTN1-AS1 promotes tumor growth of hepatocellular carcinoma by targeting miR-23c/ERBB2IP axis. Biomed Pharmacother. 2019; 109: 1140-7.

10. Wang $Y$, Sun $\mathrm{L}$, Wang L, Liu Z, Li Q, Yao B, et al. Long non-coding RNA DSCR8 acts as a molecular sponge for miR-485-5p to activate Wnt/beta-catenin signal pathway in hepatocellular carcinoma. Cell Death Dis. 2018; 9: 851

11. Sun L, Wang L, Chen T, Shi Y, Yao B, Liu Z, et al. LncRNA RUNX1-IT1 which is downregulated by hypoxia-driven histone deacetylase 3 represses proliferation and cancer stem-like properties in hepatocellular carcinoma cells. Cell Death Dis. 2020; 11: 95.

12. Sun TT, He J, Liang Q, Ren LL, Yan TT, Yu TC, et al. LncRNA GClnc1 Promotes Gastric Carcinogenesis and May Act as a Modular Scaffold of WDR5 and KAT2A Complexes to Specify the Histone Modification Pattern. Cancer Discov. 2016; 6: 784-801.

13. Tang J, Yan T, Bao Y, Shen C, Yu C, Zhu X, et al. LncRNA GLCC1 promotes colorectal carcinogenesis and glucose metabolism by stabilizing c-Myc. Nat Commun. 2019; 10: 3499.

14. Niu Y, Bao L, Chen Y, Wang C, Luo M, Zhang B, et al. HIF2-Induced Long Noncoding RNA RAB11B-AS1 Promotes Hypoxia-Mediated Angiogenesis and Breast Cancer Metastasis. Cancer Res. 2020; 80: 964-75.

15. Zhao J, Du P, Cui P, Qin Y, Hu C, Wu J, et al. LncRNA PVT1 promotes angiogenesis via activating the STAT3/VEGFA axis in gastric cancer. Oncogene. 2018; 37: 4094-109.

16. Zhang E, He X, Zhang C, Su J, Lu X, Si X, et al. A novel long noncoding RNA HOXC-AS3 mediates tumorigenesis of gastric cancer by binding to YBX1. Genome Biol. 2018; 19: 154

17. Zhao Y, Liu Y, Lin L, Huang Q, He W, Zhang S, et al. The lncRNA MACC1-AS1 promotes gastric cancer cell metabolic plasticity via AMPK/Lin28 mediated mRNA stability of MACC1. Mol Cancer. 2018; 17: 69 .

18. Sun $Y$, Wang $\mathrm{P}$, Yang $\mathrm{W}$, Shan $\mathrm{Y}$, Zhang $\mathrm{Q}$, Wu $\mathrm{H}$. The role of lncRNA MSC-AS1/miR-29b-3p axis-mediated CDK14 modulation in pancreatic cancer proliferation and Gemcitabine-induced apoptosis. Cancer Biol Ther. 2019; 20: 729-39.

19. Cao C, Zhong Q, Lu L, Huang B, Li J, Meng L, et al. Long noncoding RNA MSC-AS1 promotes hepatocellular carcinoma oncogenesis via inducing the expression of phosphoglycerate kinase 1. Cancer Med. 2020; 9: 5174-84.

20. Yao H, Yang L, Tian L, Guo Y, Li Y. LncRNA MSC-AS1 aggravates nasopharyngeal carcinoma progression by targeting miR-524-5p/nuclear receptor subfamily 4 group A member 2 (NR4A2). Cancer Cell Int. 2020; 20: 138.

21. Hu Z, Li L, Cheng P, Liu Q, Zheng X, Peng F, et al. IncRNA MSC-AS1 activates Wnt/beta-catenin signaling pathway to modulate cell proliferation and migration in kidney renal clear cell carcinoma via miR-3924/WNT5A. J Cell Biochem. 2020.

22. Yin G, Zhou H, Xue Y, Yao B, Zhao W. MicroRNA-340 promotes the tumor growth of human gastric cancer by inhibiting cyclin G2. Oncol Rep. 2016; 36: 1111-8.

23. Fan H, Jin X, Liao C, Qiao L, Zhao W. MicroRNA-301b-3p accelerates the growth of gastric cancer cells by targeting zinc finger and BTB domain containing 4. Pathol Res Pract. 2019; 215: 152667.

24. Tang Z, Li C, Kang B, Gao G, Zhang Z. GEPIA: a web server for cancer and normal gene expression profiling and interactive analyses. Nucleic Acids Res. 2017; 45: W98-W102.

25. Zheng $\mathrm{Y}, \mathrm{Xu} \mathrm{Q}$, Liu $\mathrm{M}, \mathrm{Hu} \mathrm{H}$, Xie $\mathrm{Y}, \mathrm{Zuo} \mathrm{Z}$, et al. $\operatorname{lnCAR:A}$ Comprehensive Resource for lncRNAs from Cancer Arrays. Cancer Res. 2019; 79: 2076-83.

26. Pavlova NN, Thompson CB. The Emerging Hallmarks of Cancer Metabolism. Cell Metab. 2016; 23: 27-47.

27. Choi RS, Lai WYX, Lee LTC, Wong WLC, Pei XM, Tsang HF, et al. Current and future molecular diagnostics of gastric cancer. Expert Rev Mol Diagn. 2019; 19: 863-74.

28. Lin LY, Yang L, Zeng Q, Wang L, Chen ML, Zhao ZH, et al Tumor-originated exosomal lncUEGC1 as a circulating biomarker for early-stage gastric cancer. Mol Cancer. 2018; 17: 84.

29. Sukonina V, Ma H, Zhang W, Bartesaghi S, Subhash S, Heglind M, et al. FOXK1 and FOXK2 regulate aerobic glycolysis. Nature. 2019; 566: 279-83.

30. Xu Q, Tu J, Dou C, Zhang J, Yang L, Liu X, et al. HSP90 promotes cell glycolysis, proliferation and inhibits apoptosis by regulating PKM2 abundance via Thr-328 phosphorylation in hepatocellular carcinoma. Mol Cancer. 2017; 16: 178

31. Han J, Meng Q, Xi Q, Wang H, Wu G. PFKFB3 was overexpressed in gastric cancer patients and promoted the proliferation and migration of gastric cancer cells. Cancer Biomark. 2017; 18: 249-56.

32. Zhu W, Ye L, Zhang I, Yu P, Wang H, Ye Z, et al. PFK15, a Small Molecule Inhibitor of PFKFB3, Induces Cell Cycle Arrest, Apoptosis and Inhibits Invasion in Gastric Cancer. PLoS One. 2016; 11: e0163768.

33. Shi L, Pan H, Liu Z, Xie J, Han W. Roles of PFKFB3 in cancer. Signal Transduct Target Ther. 2017; 2: 17044

34. De Bock K, Georgiadou M, Schoors S, Kuchnio A, Wong BW, Cantelmo $A R$, et al. Role of PFKFB3-driven glycolysis in vessel sprouting. Cell. 2013; 154: 651-63.

35. Domenech E, Maestre C, Esteban-Martinez L, Partida D, Pascual $R$ Fernandez-Miranda G, et al. AMPK and PFKFB3 mediate glycolysis and 
survival in response to mitophagy during mitotic arrest. Nat Cell Biol. 2015; 17: 1304-16.

36. Bobarykina AY, Minchenko DO, Opentanova IL, Moenner M, Caro J, Esumi H, et al. Hypoxic regulation of PFKFB-3 and PFKFB-4 gene expression in gastric and pancreatic cancer cell lines and expression of PFKFB genes in gastric cancers. Acta Biochim Pol. 2006; 53: 789-99. 